\title{
FITORREMEDIAÇÃO DE SOLO CONTAMINADO COM PICLORAM POR CAPIM-PÉ-DE-GALINHA-GIGANTE ${\text { (Eleusine coracana })^{(1)}}^{(1)}$
}

\author{
Sergio de Oliveira Procópio ${ }^{(2)}$, Marcos Lima do Carmo $^{(3)}$, Fábio \\ Ribeiro Pires ${ }^{(4)}$, Alberto Cargnelutti Filho ${ }^{(5)}$, Guilherme Braga \\ Pereira Braz ${ }^{(6)}$, Welington Fernando Peres Silva ${ }^{(6)}$, Alberto Leão de \\ Lemos Barroso $^{(7)}$, Gilson Pereira Silva ${ }^{(7)}$, Eduardo Lima do Carmo ${ }^{(3)}$ \\ \& Antonio Joaquim Braga Pereira Braz ${ }^{(7)}$
}

\begin{abstract}
RESUMO
O longo efeito residual do herbicida picloram no solo aumenta o risco de lixiviação e de fitotoxicidade em culturas sucedâneas; sua presença no solo pode ser abreviada com o uso da fitorremediação. $O$ objetivo deste trabalho foi avaliar a influência da densidade populacional de capim-pé-de-galinha-gigante (Eleusine coracana) sobre a fitorremediação de solo contaminado com o herbicida picloram. O experimento foi realizado em casa de vegetação localizada no município de Rio Verde-GO, no período de setembro de 2006 a fevereiro de 2007. Os tratamentos foram compostos pela combinação entre quatro densidades populacionais da espécie vegetal Eleusine coracana (capim-pé-de-galinha-gigante) (0, 7, 14 e 21 plantas por vaso, correspondendo a $0,172,344$ e 516 plantas $\mathrm{m}^{-2}$, respectivamente) e três doses do picloram $\left(0,80\right.$ e $160 \mathrm{~g}^{\mathrm{ha}^{-1}}$ - aplicadas diretamente nos vasos, simulando níveis de contaminação do solo). Após o cultivo da espécie vegetal
\end{abstract}

\footnotetext{
(1) Parte da Dissertação de Mestrado do segundo autor apresentada ao Curso de Pós-Graduação em Produção Vegetal, Departamento de Agronomia, Universidade de Rio Verde - FES/URV. Recebido para publicação em dezembro de 2007 e aprovado em outubro de 2008.

(2) Pesquisador da Embrapa Tabuleiros Costeiros. CEP 49025-040 Aracaju (SE). Bolsista do CNPq. E-mail: procopio@cpatc.embrapa.br

${ }^{(3)}$ Mestrando do Programa de Pós-Graduação da Fesurv, Universidade de Rio Verde - URV. Caixa Postal 104, CEP $75901-970$ Rio Verde (GO). E-mail: marcoslima@fesurv.br

(4) Professor do Departamento de Ciências da Saúde, Biológicas e Agrárias do Centro Universitário Norte - ES/UFES. CEP 29933480 São Mateus (ES). fabiopires@ceunes.ufes.br

(5) Professor do Departamento de Estatística, Universidade Federal do Rio Grande do Sul - UFRGS. Av. Bento Gonçalves 7712 , Caixa Postal 15100, CEP 91501-970 Porto Alegre (RS). E-mail: cargnelutti@ufrgs.br

(6) Discente de Agronomia, FES/URV. E-mail: guilhermebrag@gmail.com; welingtonperess@zipmail.com.br

(7) Professor da Faculdade de Agronomia, FES/URV. E-mails: allbarroso@fesurv.br, gilson@fesurv.br, braga@fesurv.br
} 


\begin{abstract}
fitorremediadora no substrato por 100 dias, efetuou-se, no próprio vaso, a semeadura da soja (Glycine max L.), espécie utilizada como bioindicadora da presença do picloram. A espécie Eleusine coracana mostrou ter capacidade de remediar solos contaminados com o herbicida picloram. A partir de 172 plantas $\mathrm{m}^{-2}$, aumentos na densidade populacional da espécie fitorremediadora não proporcionaram redução de carryover do herbicida picloram sobre a cultura da soja semeada em sucessão.
\end{abstract}

Termos de indexação: biorremediação, residual de herbicidas, descontaminação do solo, Glycine max.

\title{
SUMMARY: PHYTOREMEDIATION OF PICLORAM-CONTAMINATED SOIL $B Y$ Eleusine coracana
}

The long-term residual effect of the herbicide picloram poses risks of leaching and phytotoxicity to successive crops. Phytoremediation can abbreviate the long carryover effect. This research was carried out from September 2006 to February 2007, under green house conditions, in Rio Verde, Goiás, Brazil, to study the influence of Eleusine coracana population density on phytoremediation of soil contaminated with picloram. The treatments consisted of the combination of four Eleusine coracana population densities (0, 7, 14 and 21 plants per pot, corresponding to 0,172, 344 and 516 plants $\mathrm{m}^{-2}$, respectively) and three picloram rates $\left(0,80\right.$ and $160 \mathrm{~g} \mathrm{ha}^{-1}$ - applied directly to the pots, simulating soil contamination levels). After growing the phytoremediation species on substrate during 100 days, soybean was sown as a bioindicator species in the same pot. E. coracana was able to remediate soils contaminanted with picloram. At densities above 172 plants $\mathrm{m}^{-2}$, there was no further reduction of the carryover effect to soybean sown in succession.

Index terms: bioremediation, herbicide carryover, soil decontamination.

\section{INTRODUÇÃO}

Herbicidas que apresentam atividade residual no solo, impedindo ou reduzindo a emergência de plantas daninhas, são importantes insumos para a garantia da produtividade das culturas comerciais, principalmente para aquelas que apresentam extenso período total de prevenção da interferência das plantas daninhas (PTPI). Contudo, após o término do PTPI, que muitas vezes coincide com o fechamento do dossel do cultivo agrícola, a presença do herbicida no solo pode se tornar indesejável, podendo resultar em carryover (Belo et al., 2007), contaminação de mananciais de água subterrânea pela lixiviação e, ou, superficiais via runoff (Barra et al., 1999; Palma et al., 2004; Krutz et al., 2005), toxicidade a organismos não-alvo (Rousseaux et al., 2003), podendo até, dependendo da recalcitrância do composto, acumularse na cadeia alimentar (Edwards, 1973).

O herbicida picloram (ácido 4-amino 3,5,6 tricloro2-piridinacarboxílico), apesar de ser utilizado, normalmente, em pós-emergência das plantas daninhas, sobretudo de dicotiledôneas arbustivas ou arbóreas (Pinho et al., 2007) em pastagens, apresenta, em relação aos demais herbicidas registrados no Brasil, um dos maiores períodos de atividade residual em solos (Santos et al., 2006b). Essa característica impede a curto e a médio prazo o cultivo de várias espécies agrícolas em áreas onde ele tenha sido empregado. É comprovado também que, quanto maior o tempo de permanência do herbicida no solo, maior o risco de contaminação do lençol freático (Bovey \& Richardson, 1991).

Trabalhos mostram que o cultivo de determinadas espécies vegetais pode descontaminar áreas com a presença de xenobióticos, como os herbicidas de ação de solo (Pilon-Smits, 2005). Essa técnica é denominada fitorremediação (Sulmon et al., 2007) e pode abreviar o tempo de liberação da área para o plantio de espécies vegetais não-tolerantes ao composto persistente. Os mecanismos fisiológicos responsáveis pela remediação mais relatados na literatura são a fitoextração (Schnoor et al., 1995) e a fitoestimulação. $\mathrm{Na}$ fitoextração, o contaminante é absorvido pelo sistema radicular da planta remediadora, podendo ser posteriormente fitocompartimentalizado, fitovolatilizado, fitoexsudado ou fitodegradado (Susarla et al., 2002). Já na fitoestimulação a planta remediadora libera no solo compostos que estimulam determinada microbiota a metabolizar o xenobiótico (rizodegradação) (Santos et al., 2007). Ambos os mecanismos podem ser influenciados pela densidade populacional das espécies remediadoras (Santos et al., 2006a), pois o aumento no volume de exploração do sistema radicular e da 
transpiração das plantas por área pode resultar em maior absorção dos xenobióticos, além de aumentar a população dos microrganismos rizodegradadores associados às suas raízes.

Pesquisas recentes demonstraram a tolerância da espécie capim-pé-de-galinha-gigante (Eleusine coracana) ao picloram, selecionada entre mais de 20 espécies, e sua potencial capacidade de reduzir os teores de picloram no solo (Carmo et al., 2008).

Considerando então o tempo de permanência do herbicida no solo, a possibilidade de contaminação do lençol freático e a entrada na cadeia alimentar, o objetivo deste trabalho foi avaliar a influência da densidade populacional de capim-pé-de-galinha-gigante (Eleusine coracana) sobre a fitorremediação de solo contaminado com o herbicida picloram.

\section{MATERIAL E MÉTODOS}

O experimento foi realizado em casa de vegetação da Fesurv - Universidade de Rio Verde, localizada no município de Rio Verde-GO. O período de condução do experimento foi de setembro de 2006 a fevereiro de 2007.

Os tratamentos foram compostos pela combinação entre quatro densidades populacionais da espécie vegetal Eleusine coracana - capim-pé-de-galinhagigante $(0,7,14$ e 21 plantas por vaso com área média de $0,0408 \mathrm{~m}^{2}$, correspondendo a 0, 172, 344 e 516 plantas $\mathrm{m}^{-2}$, respectivamente) e três doses do picloram $\left(0,80\right.$ e $\left.160 \mathrm{~g} \mathrm{ha}^{-1}\right)$, totalizando 12 tratamentos. O delineamento experimental utilizado foi o inteiramente casualizado em esquema fatorial 4 x 3, com quatro repetições. As densidades populacionais utilizadas correspondem a zero, uma, duas e três vezes a recomendação por hectare para essa espécie, levando-se em consideração o valor cultural da semente. Já as doses de picloram correspondem a zero, um quarto e metade da dose do herbicida Tordon ${ }^{\circledR}$ recomendada por hectare para pastagem, definidas com base no trabalho de seleção de espécies tolerantes ao picloram (Carmo et al., 2008).

Como substrato para o crescimento das plantas, utilizaram-se amostras de solo classificado como Latossolo Vermelho eutroférrico (Quadro 1). O solo foi coletado na camada de $0-20 \mathrm{~cm}$, em área homogênea de Cerrado nativo pertencente à FESURV, não cultivado e, portanto, sem histórico de aplicação de herbicidas.

Antes do preenchimento dos vasos com capacidade para $8 \mathrm{dm}^{3}$, o solo foi corrigido com calcário filler, utilizando-se o equivalente a $2 \mathrm{t} \mathrm{ha}^{-1}$, e adubado com $500 \mathrm{~kg} \mathrm{ha}{ }^{-1}$ da fórmula 08-20-18. Essas quantidades foram calculadas com base na análise de solo, visando atender às necessidades nutricionais médias de gramíneas forrageiras (Sousa \& Lobato, 2004), já que não existe uma recomendação específica para a espécie E. coracana.

Quarenta e oito horas após o preenchimento dos vasos e umedecimento do solo até a capacidade de campo, procedeu-se à aplicação do herbicida picloram, utilizando-se um pulverizador costal pressurizado com $\mathrm{CO}_{2}$, acoplado de barra contendo duas pontas de pulverização TT 110.02, aplicando volume de calda equivalente a $200 \mathrm{~L} \mathrm{ha}^{-1}$. Após $48 \mathrm{~h}$ da aplicação do herbicida, foi realizada a semeadura de capim-pé-degalinha-gigante, espécie fitorremediadora. Dez dias após a emergência das plantas, realizou-se um desbaste, deixando-se o número de plantas por vaso correspondente a cada tratamento. Todos os vasos foram irrigados duas vezes ao dia, para manutenção da umidade do solo, tomando-se como referência a média da água evaporada, determinada por meio de dois evaporímetros (recipientes instalados dentro da área experimental com área de borda de mesma dimensão dos vasos usados para plantio das espécies).

Decorridos 100 dias após a emergência da espécie fitoremediadora, as plantas foram dessecadas, com glyphosate (1.800 $\mathrm{g} \mathrm{ha}^{-1}$ de equivalente ácido); cinco dias após a dessecação as plantas foram cortadas na

\section{Quadro 1. Atributos químicos e textura do solo utilizado no experimento}

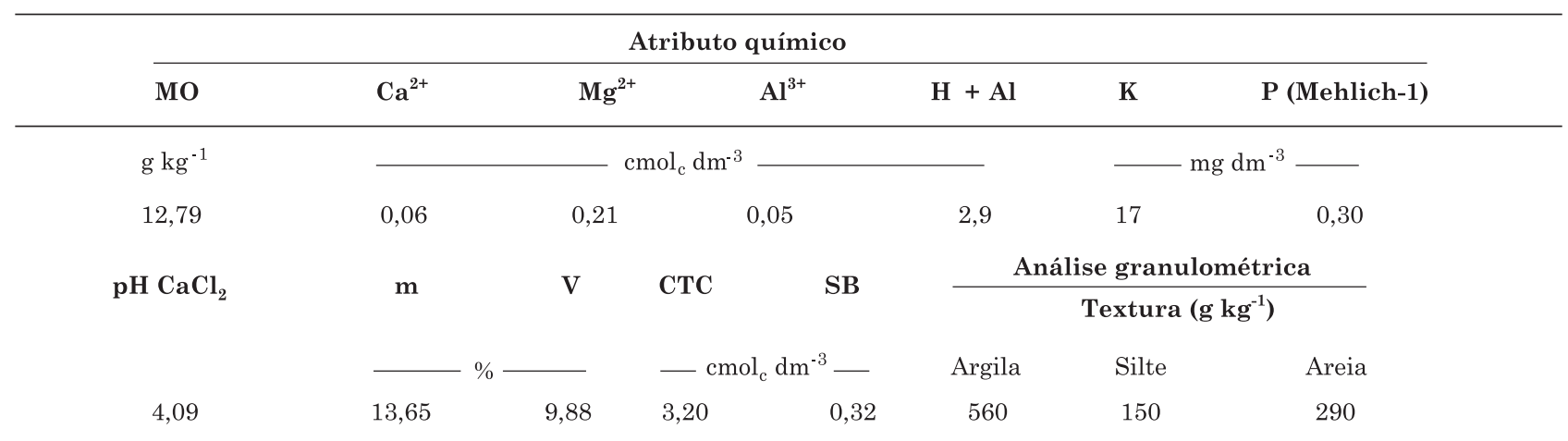

Análises realizadas nos Laboratórios de Análises Físicas e Químicas de Solo da Faculdade de Agronomia da Fesurv - Universidade de Rio Verde, segundo a método descrito pela Empresa Brasileira de Pesquisa Agropecuária-Embrapa (1997). 
altura do coleto e a parte aérea, descartada. A seguir, a fim de uniformizar a disponibilidade de nutrientes em cada unidade experimental para a etapa seguinte (bioensaio), foi retirada uma amostra de solo de cada vaso, utilizando-se uma sonda de $2 \mathrm{~cm}$ de diâmetro, que penetrava da borda até a base do vaso. Essas amostras foram homogeneizadas por tratamento (combinação entre dose e densidade populacional) e analisadas quanto aos teores de macro e micronutrientes (Embrapa, 1997). De posse dos resultados, procedeuse à adubação de plantio específica para cada tratamento, fornecendo os nutrientes via líquida, na superfície dos vasos, sem revolvimento do solo. Terminada esta etapa, efetuou-se a semeadura da espécie bioindicadora da presença do picloram - soja (Glycine max cultivar Monsoy 6101), distribuindo-se 10 sementes por vaso. Após a emergência das plantas da espécie bioindicadora, procedeu-se a desbaste, deixando-se três plantas por vaso. Novamente, como na etapa anterior, todos os vasos foram irrigados duas vezes ao dia.

Aos 15 e 40 dias após a emergência (DAE) das plantas bioindicadoras, avaliou-se a fitotoxicidade visualmente utilizando-se escala percentual, em que 0 (zero) significa ausência de sintomas (epinastia das plantas, diminuição da área foliar e encarquilhamento das folhas, paralisação do crescimento) e $100 \%$, morte de todas as plantas. A altura das plantas foi determinada por escala graduada, tendo como referência o meristema apical. Aos 40 DAE, as plantas bioindicadoras foram cortadas rente ao solo, sendo o material vegetal imediatamente colocado em estufa de circulação forçada de ar $\left(70 \pm 2^{\circ} \mathrm{C}\right)$ por $72 \mathrm{~h}$ e pesado, determinando-se assim a massa da parte aérea seca. Esses procedimentos foram realizados conforme Santos et al. (2006a).

Após a coleta e tabulação dos dados, estes foram submetidos à análise de variância. As médias dos efeitos significativos das doses do picloram, em cada densidade populacional, foram comparados pelo teste de Tukey a $5 \%$, devido ao número insuficiente de níveis para o ajuste das equações de regressão.

\section{RESULTADOS E DISCUSSÃO}

Quando não se efetuou o cultivo prévio das plantas de E. coracana, as injúrias observadas nas plantas de soja, causadas pelo herbicida picloram, foram severas (Quadro 2), ratificando a soja como uma espécie muito sensível à presença do picloram no solo, mesmo em quantidades extremamente baixas (Wax et al., 1969). Essa constatação reforça o cuidado com o plantio dessa leguminosa em áreas onde esse herbicida tenha sido empregado anteriormente.

O cultivo prévio de $E$. coracana nas três densidades populacionais $\left(172,344\right.$ e 516 plantas $\left.\mathrm{m}^{-2}\right)$ reduziu significativamente a intoxicação das plantas de soja avaliadas aos 15 DAE (abaixo de $12 \%$ ), quando o nível de contaminação inicial era de 80 ou $160 \mathrm{~g} \mathrm{ha}^{-1} \mathrm{de}$ picloram (Quadro 2). Todavia, a partir da densidade de 172 plantas $\mathrm{m}^{-2}$, as densidades populacionais de $E$. coracana testadas não influenciaram a eficácia da técnica remediadora. O mesmo comportamento foi observado aos 40 DAE, porém os níveis de intoxicação em todos os tratamentos foram mais acentuados que na primeira avaliação. Apesar disso, a utilização de 172 plantas $\mathrm{m}^{-2}$ proporcionou diminuição considerável nos níveis de intoxicação das plantas de soja - em média, inferiores a $65 \%$, quando comparados aos tratamentos sem fitorremediação, com injúrias acima de $97 \%$ (plantas praticamente mortas pela atividade residual do picloram) (Quadro 2). A intensificação da fitotoxicidade aos 40 DAE em relação à primeira avaliação pode ser atribuída ao maior tempo de

Quadro 2. Fitotoxicidade em plantas de soja semeadas após o cultivo prévio de Eleusine coracana (capimpé-de-galinha-gigante), em quatro densidades populacionais, em solo contaminado com três níveis do herbicida picloram

\begin{tabular}{|c|c|c|c|c|}
\hline \multirow{2}{*}{ Dose de picloram } & \multicolumn{4}{|c|}{ Planta de Eleusine coracana $\mathrm{m}^{-2}$} \\
\hline & $\mathbf{0}$ & 172 & 344 & 516 \\
\hline $\mathrm{g} \mathrm{ha}^{-1}$ & \multicolumn{4}{|c|}{ Fitotoxicidade (\%) em plantas de soja aos $15 \mathrm{DAE}^{(1)}$} \\
\hline 0 & $0,00 \mathrm{~b}$ & $0,00 \mathrm{~b}$ & $0,00 \mathrm{~b}$ & $0,00 \mathrm{~b}$ \\
\hline 80 & 92,25 a & $6,00 \mathrm{a}$ & 7,25 a & $10,25 \mathrm{a}$ \\
\hline 160 & 94,75 a & $7,25 \mathrm{a}$ & $11,75 \mathrm{a}$ & $10,50 \mathrm{a}$ \\
\hline \multicolumn{5}{|c|}{ Fitotoxicidade (\%) em plantas de soja aos $40 \mathrm{DAE}$} \\
\hline 0 & $0,00 \mathrm{~b}$ & $0,00 \mathrm{~b}$ & $0,00 \mathrm{~b}$ & $0,00 \mathrm{~b}$ \\
\hline 80 & 99,75 a & 49,75 a & 56,75 a & $57,00 \mathrm{a}$ \\
\hline 160 & $97,25 \mathrm{a}$ & $58,75 \mathrm{a}$ & $64,50 \mathrm{a}$ & $62,50 \mathrm{a}$ \\
\hline
\end{tabular}

(1) DAE: dias após a emergência. Médias não seguidas de mesma letra na vertical diferem pelo teste de Tukey a $5 \%$. 
exposição ao herbicida no solo e à maior absorção pela soja, pois, à medida que a planta cresce, o sistema radicular explora maior volume de solo, aumentando a absorção do herbicida. Esse comportamento foi também verificado por Carmo et al. (2008), trabalhando com picloram, e Pires et al. (2003), com o herbicida tebuthiuron.

A densidade adequada dependerá primordialmente da espécie utilizada, porém, de acordo com Schnoor \& Dee (1997), que trabalharam com espécies arbóreas como agentes fitorremediadores, a alta densidade populacional no início do estabelecimento das plantas assegura significativa taxa evapotranspirativa, o que é desejável até certo ponto, pois, com o aumento demasiado da densidade, a competição intra-específica pode prejudicar o crescimento e o desenvolvimento das plantas.

Segundo Santos et al. (2006a), aumentos da densidade populacional de plantas remediadoras em determinada área, até certo limite, também podem proporcionar maior volume de raízes e de solo explorado, podendo resultar em incremento da absorção/degradação do contaminante e, ou, degradação rizosférica. Portanto, era de se esperar que, com o aumento da densidade, houvesse redução da fitotoxicidade, decorrente do incremento na fitorremediação promovida pelo E. coracana. Todavia, neste trabalho, esse efeito não foi observado. A competição intra-específica pode ser a causa do nãoaumento na eficiência da fitorremediação pelo incremento na densidade populacional de $E$. coracana, uma vez que o excesso de plantas pode acarretar limitação da exploração do solo, com conseqüente estabilização da interceptação/remoção do herbicida pelo sistema radicular das plantas de E. coracana.

O plantio de E. coracana parece ter estimulado o crescimento das plantas de soja semeadas em sucessão, porque, mesmo não tendo sido comparada estatisticamente, em valores absolutos, a média de altura de plantas foi superior em solo sem herbicida onde foi cultivada essa espécie, em comparação com a testemunha sem plantas. Esse fato foi constatado apenas na avaliação realizada aos 40 DAE (Quadro 3), o que mostra mais um possível benefício da utilização dessa espécie forrageira em programas de fitorremediação que visem à introdução nas áreas de cultivo da soja.

A altura das plantas de soja decresceu significativamente aos 15 e $40 \mathrm{DAE}$, quando a cultura foi cultivada em solo com a presença do picloram nos dois níveis iniciais de contaminação (80 e $\left.160 \mathrm{~g} \mathrm{ha}^{-1}\right)$ e sem o cultivo prévio de E. coracana. Aos 40 DAE, a altura média das plantas de soja que cresceram em solo sem herbicida foi de $28,95 \mathrm{~cm}$, enquanto nos solos contaminados as plantas não atingiram, em média, $2 \mathrm{~cm}$ (Quadro 3). Scifres et al. (1972) relatam que plantas de feijão e girassol podem exibir sintomas de intoxicação quando cultivadas em solos que contêm menos de $10 \mathrm{ppb}$ de picloram. Aos $15 \mathrm{DAE}$, a implementação da fitorremediação com plantas de $E$. coracana, nas três densidades avaliadas $(172,344 \mathrm{e}$ 516 plantas $\mathrm{m}^{-2}$ ), impediu a redução do porte das plantas de soja, mesmo no maior nível de contaminação do solo (160 $\mathrm{g} \mathrm{ha}^{-1}$ de picloram) (Quadro 3). Apesar disso, não foi suficiente para impedir a redução na altura das plantas de soja aos 40 DAE (Quadro 3).

Nota-se também intensificação na redução do porte das plantas de soja, em relação à testemunha, nas doses de 80 e $160 \mathrm{~g} \mathrm{ha}^{-1}$ de picloram aos 40 DAE, em comparação com o observado aos 15 DAE (Quadro 3). Esse comportamento condiz com o observado por Pires et al. (2003) e, provavelmente, se deve à maior ação fitotóxica do composto herbicida absorvido ao longo do período de crescimento da planta no solo contaminado.

Quadro 3. Altura de plantas de soja semeadas após o cultivo prévio de Eleusine coracana (capim-pé-degalinha-gigante), em quatro densidades populacionais, em solo contaminado com três níveis do herbicida picloram

\begin{tabular}{|c|c|c|c|c|}
\hline \multirow{2}{*}{ Dose de picloram } & \multicolumn{4}{|c|}{ Planta de Eleusine coracana $\mathrm{m}^{-2}$} \\
\hline & 0 & 172 & 344 & 516 \\
\hline $\mathrm{g} \mathrm{ha}^{-1}$ & \multicolumn{4}{|c|}{ Altura de plantas de soja $(\mathrm{cm})$ aos $15 \mathrm{DAE}^{(1)}$} \\
\hline 0 & $11,98 \mathrm{a}$ & $12,28 \mathrm{a}$ & $12,00 \mathrm{a}$ & $12,70 \mathrm{a}$ \\
\hline 80 & $4,90 \mathrm{~b}$ & $13,43 \mathrm{a}$ & $13,10 \mathrm{a}$ & $12,63 \mathrm{a}$ \\
\hline \multirow[t]{2}{*}{160} & $4,68 \mathrm{~b}$ & $12,38 \mathrm{a}$ & $13,10 \mathrm{a}$ & $12,10 \mathrm{a}$ \\
\hline & \multicolumn{4}{|c|}{ Altura de plantas de soja $(\mathrm{cm})$ aos 40 DAE } \\
\hline 0 & $28,95 \mathrm{a}$ & $40,48 \mathrm{a}$ & $43,20 \mathrm{a}$ & $41,90 \mathrm{a}$ \\
\hline 80 & $0,58 \mathrm{~b}$ & $30,13 \mathrm{~b}$ & $32,98 \mathrm{~b}$ & $27,55 \mathrm{~b}$ \\
\hline 160 & $1,33 \mathrm{~b}$ & $28,00 \mathrm{~b}$ & $27,80 \mathrm{~b}$ & $29,55 \mathrm{~b}$ \\
\hline
\end{tabular}

(1) DAE: dias após a emergência. Médias não seguidas de mesma letra na vertical diferem pelo teste de Tukey a 5 \%. 
A avaliação da altura das plantas bioindicadoras mostrou novamente a tendência de estabilização da eficiência da fitorremediação com o aumento da densidade das plantas remediadoras, a partir de, aproximadamente, 172 plantas $\mathrm{m}^{-2}$ de E. coracana (Quadro 3), fato que ficou evidente nos dois níveis iniciais de contaminação (80 ou $160 \mathrm{~g} \mathrm{ha}^{-1}$ de picloram). De acordo com Lindsay et al. (2003), comumente utiliza-se densidade maior que aquela recomendada para o cultivo comercial das espécies, quando se trata de espécie cultivada com o objetivo de fitorremediação de uma área, particularmente se a espécie não é totalmente conhecida. No caso de E. coracana, uma espécie relativamente estudada e cultivada, os resultados apontam para a utilização da densidade comercial já recomendada, o que é interessante por não onerar os custos referentes à aquisição de sementes no programa de remediação com essa planta.

O acúmulo de massa por plantas de soja foi favorecido pelo cultivo anterior de plantas de $E$. coracana, independentemente da presença ou não do picloram no solo, reforçando outros benefícios advindos do cultivo prévio dessa forrageira que não somente a ação fitorremediadora, como a ciclagem de nutrientes e a melhor estruturação do solo, sobretudo no caso de gramíneas (Quadro 4). A atividade residual do picloram no solo resultou em diminuição drástica da massa da parte aérea seca das plantas de soja aos $40 \mathrm{DAE}$, quando não foi realizada a fitorremediação do solo com as plantas de E. coracana (Quadro 4). Todas as densidades populacionais avaliadas de $E$. coracana $\left(172,344\right.$ e 516 plantas $\mathrm{m}^{-2}$ ) foram capazes de garantir maior acúmulo de massa da parte aérea seca das plantas bioindicadoras quando estas foram cultivadas em solo contaminado com 80 ou $160 \mathrm{~g} \mathrm{ha}^{-1}$ de picloram. Ainda assim, nenhum tratamento fitorremediador promoveu o mesmo acúmulo de massa na parte aérea das plantas de soja, em comparação com os tratamentos sem a contaminação prévia do picloram (Quadro 4). A partir de 172 plantas $\mathrm{m}^{-2}$ de E. coracana, o aumento da população dessa espécie não acarretou evolução da eficácia dessa técnica descontaminadora de solos com esse xenobiótico (Quadro 4). Santos et al. (2006a) verificaram que a densidade populacional mínima da espécie fitorremediadora Canavalia ensiformis, que possibilitou o melhor crescimento e a máxima produtividade do feijoeiro cultivado em solo contaminado com o herbicida trifloxysulfuron-sodium, foi de 20 plantas $\mathrm{m}^{-2}$. Segundo Procópio et al. (2005), plantas de mucuna-preta (Stizolobium aterrimum) foram eficientes na remediação do herbicida trifloxysulfuron-sodium, constatando que a densidade populacional mínima desse adubo verde deve ser de 25 plantas $\mathrm{m}^{-2}$. Nesses dois trabalhos, as densidades populacionais indicadas correspondem a 2,5 vezes aquela recomendada na adubação verde.

A possibilidade de utilização de E. coracana como espécie fitorremediadora mostra-se promissora também porque ela apresenta baixo risco de se tornar uma espécie daninha, pois, além do efeito remediador, da facilidade de multiplicação via sementes (quando for o caso) e do baixo custo de aquisição, seu controle posterior é simples, por se tratar de uma espécie anual e com alta sensibilidade ao herbicida glyphosate.

Um aspecto que deve ser considerado é que neste trabalho procedeu-se à semeadura da espécie remediadora pouco tempo após a aplicação do herbicida picloram, não considerando um possível período total de prevenção à interferência (PTPI) das plantas daninhas. Essa informação é importante para a implementação de um programa de fitorremediação quando se trata de culturas agrícolas, pois, com o tempo, a interação do pesticida com o solo sofre um "envelhecimento" (aging), tornando-se o pesticida mais resistente à extração e aumentando sua concentração na forma de resíduo ligado. Contudo, para pastagem, que é o alvo principal das aplicações de picloram, o conceito de PTPI não se aplica, já que a questão-chave é saber quando a pastagem que recebeu a aplicação do picloram será transformada em lavoura, principalmente de espécies dicotiledôneas, como soja, tomate, feijão, algodão, etc. Desse modo, a necessidade de fitorremediação em áreas de pastagens pode se dar a qualquer momento após a aplicação, sejam dias ou anos, uma vez que o picloram pode permanecer ativo no solo por muito tempo (Bovey \& Richardson, 1991). Se, por um lado, a concentração do herbicida diminui

Quadro 4. Massa de plantas de soja seca semeadas após o cultivo prévio de Eleusine coracana (capim-pé-degalinha-gigante), em quatro densidades populacionais, em solo contaminado com três níveis do herbicida picloram

\begin{tabular}{ccccc}
\hline \multirow{2}{*}{ Dose de picloram } & \multicolumn{4}{c}{ Planta de Eleusine coracana $\mathbf{~ m}^{-\mathbf{2}}$} \\
\cline { 2 - 4 } & \multicolumn{1}{c}{$\mathbf{0}$} & $\mathbf{1 7 2}$ & $\mathbf{3 4 4}$ & $\mathbf{5 1 6}$ \\
\hline $\mathrm{g} \mathrm{ha}^{-1}$ & \multicolumn{2}{c}{ Massa seca da parte aérea de plantas de soja (g) aos 40 DAE $^{(1)}$} \\
0 & $12,83 \mathrm{a}$ & $23,18 \mathrm{a}$ & $23,58 \mathrm{a}$ & $26,15 \mathrm{a}$ \\
80 & $3,30 \mathrm{~b}$ & $16,38 \mathrm{~b}$ & $16,10 \mathrm{~b}$ & $15,80 \mathrm{~b}$ \\
160 & $2,23 \mathrm{~b}$ & $15,80 \mathrm{~b}$ & $14,35 \mathrm{~b}$ & $15,29 \mathrm{~b}$ \\
\hline
\end{tabular}

(1) DAE: dias após a emergência. Médias não seguidas de mesma letra na vertical diferem pelo teste de Tukey a $5 \%$. 
com o tempo, reduzindo o percentual que poderia ser fitorremediado, também é certo que a sua fitotoxicidade a espécies sensíveis seria reduzida (e também seu risco de lixiviação). No entanto, pela observação dos resultados de fitotoxicidade, altura e biomassa para o tratamento testemunha (sem plantas remediadoras), verifica-se que, mesmo após 100 dias da aplicação dos tratamentos, quando se introduziu a soja como planta indicadora, o herbicida ainda permanecia em concentrações suficientes no solo para ser absorvido pelas plantas de soja e causar-lhes injúrias. Portanto, mesmo após a permanência do picloram no solo por determinado período, com possíveis interações com os colóides minerais e, ou, orgânicos, e provável maior resistência à extração, a concentração do herbicida no solo era ainda suficiente para ser absorvido e ser fitorremediado por E. coracana. Logo, as condições deste experimento validam trabalhos em que a técnica de fitorremediação é empregada próxima ao momento em que houve a contaminação do substrato.

\section{CONCLUSÕES}

1. Eleusine coracana mostrou ter capacidade de remediar solos contaminados com o herbicida picloram.

2. A partir de 172 plantas $\mathrm{m}^{-2}$, aumentos na densidade populacional da espécie fitorremediadora $E$. coracana não proporcionaram redução de carryover do herbicida picloram sobre a cultura da soja semeada em sucessão.

3. A densidade populacional de 172 plantas $\mathrm{m}^{-2}$ é a mais indicada.

\section{LITERATURA CITADA}

BARRA, R.; NOTARIANNI, V.; MAFFIOLI, G.; MAZZUCHELLI, P. \& VIGHI, M. Patrones de contaminación por herbicidas en aguas superficiales en una cuenca agrícola. Ecotox. Environ. Restoration, 2:75-83, 1999.

BELO, A.F. SANTOS, E.A.; SANTOS, J.B.; FERREIRA, L.R.; SILVA, A.A.; CECON, P.R. \& SILVA, L.L. Fitorremediação de solo adubado com composto orgânico e contaminado com trifloxysulfuron-sodium. Planta Daninha, 25:251-258, 2007.

BOVEY, R.W. \& RICHARDSON, C.W. Dissipation of clopyralid and picloram in soil and seep flow in the blacklands of Texas. J. Environ. Qual., 20:528-531, 1991.

CARMO, M.L.; PROCOPIO, S.O.; PIRES, F.R.; CARGNELUTTI FILHO, A.; BARROSO, A.L.L.; SILVA, G.P.; CARMO, E.L.; BRAZ, G.B.P.; SILVA, W.F.P.; BRAZ, A.J.B.P. \& PACHECO, L.P. Seleção de plantas para fitorremediação de solos contaminados com picloram. Planta Daninha, 26:301-313, 2008.

EDWARDS, C.A. Persistent pesticides in the environment. 2.ed. New York, CRC Press, 1973. 170p.
EMPRESA BRASILEIRA DE PESQUISA AGROPECUÁRIA EMBRAPA. Centro Nacional de Pesquisa de Solos. Manual de métodos de análise de solo. 2.ed. Rio de Janeiro, 1997. 212p.

KRUTZ, L.J.; SENSEMAN, S.A.; ZABLOTOWICZ, R.M. \& MATOCHA, M.A. Reducing herbicide runoff from agricultural fields with vegetative filter strips: A review. Weed Sci., 53:353-367, 2005.

LINDSAY, E.B.; BURKHEAD, J.L.; HALE, K.L.; TERRY, N.; PILON, M. \& PILON-SMITS, E.A.H. Analysis of transgenic indian mustard plants for phytoremediation of metal-contaminated mine tailings. J. Environ. Qual., 32:432-440, 2003

PALMA, G.; SANCHEZ, A.; OLAVE, Y.; ENCINA, F.; PALMA, R. \& BARRA, R. Pesticides levels in surfaces waters in an agricultural-forestry basin in Southern Chile. Chemosphere, 57:763-770, 2004.

PILON-SMITS, E.A.H. Phytoremediation. Ann. Rev.Plant Biol., 56:15-39, 2005.

PINHO, A.P.; MATOS, A.T.; MORRIS, L.A. \& COSTA, L.M. Atrazine and picloram adsorption in organic horizon forest samples under laboratory conditions. Planta Daninha, 25:125-131, 2007.

PIRES, F.R.; SOUZA, C.M.; SILVA, A.A.; PROCÓPIO, S.O. \& FERREIRA, L.R. Seleção de plantas com potencial para fitorremediação de tebuthiuron. Planta Daninha, 21:451$458,2003$.

PROCÓPIO, S.O.; SANTOS, J.B.; PIRES, F.R.; SILVA, A.A.; SANTOS, E.A. \& FERREIRA, L.R. Fitorremediação de solo contaminado com trifloxysulfuron sodium por mucuna-preta (Stizolobium aterrimum). Planta Daninha, 23:719-724, 2005.

ROUSSEAUX, S.; HARTMANN. A.; ROUARD, N. \& SOULAS, G. A simplified procedure for terminal restriction fragment length polymorphism analysis of the soil bacterial community to study the effects of pesticides on the soil microflore using 4,6-dinitroorthocresol as test case. Biol. Fert. Soils, 37:250-254, 2003.

SANTOS, E.A.; SANTOS, J.B.; FERREIRA, L.R.; COSTA, M.D. \& SILVA, A.A. Fitoestimulação por Stizolobium aterrimum como processo de remediação de solo contaminado com trifloxysulfuron sodium. Planta Daninha, 25:259-265, 2007.

SANTOS, J.B.; PROCÓPIO, S.O.; PIRES, F.R.; SILVA, A.A. \& SANTOS, E.A. Fitorremediação de solo contaminado com trifloxysulfuron-sodium por diferentes densidades populacionais de feijão-de-porco (Canavalia ensiformis (l). dc.). Ci. Agrotecnol., 30:444-449, 2006a.

SANTOS, M.V.; FREITAS, F.C.L.; FERREIRA, F.A.; VIANA, R.G.; TUFFI SANTOS, L.D. \& FONSECA, D.M. Eficácia e persistência no solo de herbicidas utilizados em pastagem. Planta Daninha, 24:391-398, 2006b.

SCHNOOR, J.L.; LICHT, L.A.; MCCUTCHEON, S.C.; WOLFE, N.L. \& CARREIRA, L.H. Phytoremediation of organic and nutrient contaminants. Environ. Sci. Technol., 29:318323,1995 . 
SCHNOOR, J.L. \& DEE, P.E. Technology evaluation report: phytoremediation. Pittsburgh, Ground-Water Remediation Technologies Analysis Center, 1997. 37p. (E Series: GWRTAC TE-98-01)

SCIFRES, C.J.; BOVEY, R.W. \& MERKLE, M.G. Variation in bioassay attributes as quantitative indices of picloram in soils. Weed Res., 12:8-64, 1972.

SOUSA, D.M.G. \& LOBATO, E. Cerrado: Correção do solo e adubação. 2.ed. Brasília, Embrapa Informação Tecnológica, 2004. 416p.
SUlmon, C.; GOUESBET, G.; BINET, F.; MARTINLAURENT, F.; AMRANI, A.E. \& COUÉE, I. Sucrose amendment enhances phytoaccumulation of the herbicide atrazine in Arabidopsis thaliana. Environ. Poll., 145:507-515, 2007.

SUSARLA, S.; MEDINA, V.F. \& MCCUTCHEON, S.C. Phytoremediation: An ecological solution to organic chemical contamination. Ecol. Eng., 18:647-658, 2002.

WAX, L.M.; KNUTH, L.A. \& SLIFE, F.W. Response of soybeans to 2,4-D, dicamba and picloram. Weed Sci., 17:388-393, 1969. 